This article is licensed under the Creative Commons Attribution-NonCommercialNoDerivatives 4.0 International License (CC BY-NC-ND) (http://www.karger.com/Services/OpenAccessLicense). Usage and distribution for commercial purposes as well as any distribution of modified material requires written permission.

\title{
Swift Spontaneous Regression of a Pediatric Traumatic Acute Subdural Hematoma
}

\author{
Hille Koppen ${ }^{a}$ e Enrico Arkink ${ }^{b} \quad$ Anna Nordbeck ${ }^{a}$ Lilian Sie $^{c}$ \\ Niels van der Gaag ${ }^{d}$ \\ aHagaziekenhuis, Department of Neurology, The Hague, The Netherlands; \\ bLandspitali University Hospital, Department of Radiology, Reykjavik, Iceland; \\ 'Hagaziekenhuis, Department of Pediatric Neurology, The Hague, The Netherlands; \\ dHagaziekenhuis, Department of Neurosurgery, The Hague, The Netherlands; \\ eLeiden University Medical Center, Department of Neurology, Leiden, The Netherlands
}

\section{Keywords}

Acute subdural hematoma $\cdot$ Children $\cdot$ Trauma $\cdot$ Operation

\begin{abstract}
We report the case of a 4-year-old girl with acute subdural hematoma who presented to the emergency department after an unwitnessed fall of the balcony. The hematoma was hyperdense along the left convexity of $9 \mathrm{~mm}$ thickness with a consequent mass effect with obliteration of the adjacent sulci, left lateral ventricle compression and a midline shift of $7 \mathrm{~mm}$. During her stay in the emergency department while waiting for transfer to the children intensive care unit elsewhere she slightly deteriorated neurologically. Repeat CT scan of the brain $4 \mathrm{~h}$ after initial presentation remarkably showed that the subdural hematoma had now largely disappeared, with a decrease in volume and density. Consequently, the mass effect diminished with a near normalization of the midline shift.




\section{Introduction}

An acute subdural hematoma (ASDH) is a rapidly clotting blood collection below the inner layer of the dura but external to the brain and arachnoid membrane. Mortality rate of an ASDH in the setting of major head trauma is estimated between 50 and $90 \%$ [1]. Treatment of an ASDH is based on neurologic condition and imaging findings. It consists of correction of coagulopathy if present in order to lessen the risk of hematoma expansion and evaluation if emergent surgical evacuation is warranted. Generally, evacuation of an ASDH is recommended if the hematoma exceeds $10 \mathrm{~mm}$ or the midline shift is greater than $5 \mathrm{~mm}$, regardless of the neurologic condition [2]. Nonoperative management is a viable option in less severe cases. Generally, an ASDH is thought to take weeks to months to resolve spontaneously [3]. However, spontaneous fast resolving ASDHs have also been described in the literature and are still poorly understood $[4,5]$.

The present case of a child illustrates a rapidly spontaneously resolving ASDH. This case is unique as regression of the ASDH was almost complete and observed radiologically within only a few hours, whereas her clinical situation developed in the other direction as she at that time deteriorated.

\section{Case Report}

A 4-year-old girl was admitted to the emergency department of our general hospital by her father, after being found outside on the ground floor of their first-floor apartment. The actual fall had not been witnessed, but very likely she had climbed over the fence of the balcony and fell. She did not have any medical history and did not use medication.

At presentation, the patient was weepy and complained of pain, which she could not further specify. At physical examination, the airway was open, with a respiration rate of 22 breaths per minute and a blood pressure of $134 / 85 \mathrm{~mm} \mathrm{Hg}$, pulse $113 / \mathrm{min}$. There was no external subcutaneous hematoma or laceration. Neurological examination showed a maximal Glasgow Coma Scale (GCS, E4M6V5), but alertness fluctuated. The pupils were equal and responsive. To relief the pain, $1 \mathrm{mg}$ morphine was administered intravenously. For the high fall and fluctuating alertness, we performed a nonenhanced computed tomography (CT) scan of the brain in line with our local/national guideline. It showed a hyperdense ASDH along the left convexity of $9 \mathrm{~mm}$ thickness with a consequent mass effect with obliteration of the adjacent sulci, left lateral ventricle compression and a midline shift of $7 \mathrm{~mm}$ (Fig. 1a-c). There was a thin band of low density on the inner margin of the hematoma, possibly representing cerebrospinal fluid (CSF) in the subdural space from an arachnoid tear (Fig. 1b, c).

No skull fracture was seen. In addition, a chest X-ray revealed a limited right-sided pneumothorax without evidence of any rib fractures.

Despite the radiological findings, we decided, considering her good GCS score on admission, to initiate a conservative approach with close monitoring on an intensive care unit. Due to logistics, the transfer to a collaborating academic center was delayed, and within $4 \mathrm{~h}$ after initial presentation her GSC score slightly deteriorated to E3M6V3 with normal pupil reflexes. Repeat CT scan of the brain (Fig. 1d-f) was performed which remarkably showed that the subdural hematoma had now largely disappeared, with a decrease in volume and density. Consequently, the mass effect diminished with a near normalization of the midline shift. In the posterior fossa, there was an increase in density of the CSF in the basal cisterns, the cisterna 
magna and the fourth ventricle, giving the erroneous idea of swelling of the posterior fossa. No increase in CSF density was seen in lateral ventricles and the third ventricle.

Conservative treatment was continued without specific medication and she was admitted to the pediatric intensive care department with close monitoring. In the following days, her consciousness improved spontaneously, and she could be discharged from our hospital in good health 7 days after the initial trauma.

\section{Discussion}

Guidelines on treatment and decisions on medical or neurosurgical interventions for pediatric traumatic ASDH are commonly derived from treatment protocols and literature in adults [6]. However, there are important differences between children and elderly with ASDH. Most elderly with an ASDH only have minor head injury, and the frequency of simultaneous primary brain injury is lower [7]. The present case of spontaneous regression in a young child is highly interesting, particularly for the available short interval between CT scans.

The phenomenon of spontaneous regression of an ASDH as observed by CT scan has been reported for the first time in 1986 [5, 8] and several cases have been described ever since.

Sometimes this spontaneous regression of the ASDH was observed in patients in whom conservative treatment was chosen because of a low GCS score and poor premorbid conditions [9]. Rapidly resolving ASDH (defined as 50\% reduction in volume within $72 \mathrm{~h}$ ) was reported in $19 \%$ of nonoperated patients in the age range between 19 and 102 years in a recent large retrospective study [4].

Only a few cases of spontaneous regression have been reported in children. All of them had much longer inter-CT intervals compared to our patient. A girl with an ASDH described by Nagao et al. [8] was nearly 4 years old and after being stuporous with a fixed and dilated pupil she spontaneously recovered, and craniotomy was deferred. Follow-up CT was done $48 \mathrm{~h}$ after the initial CT and showed complete resolution of the ASDH. Huang et al. [10] described a 23-month-old patient with an ASDH with spontaneous resolution on CT follow-up after $65 \mathrm{~h}$. Neurological deficit was only minor at presentation with preserved consciousness. The initial CT also showed a thin low-density area within the hematoma consistent with CSF in the subdural space, also present in our patient. In our case, the clinical deterioration was not due to increasing ASDH volume as suspected, but possibly due to transient increasing intracranial pressure, considering the isointense blood-filled basal cisterns as observed on the second CT.

Several mechanisms which could explain rapid resolution of ASDH have been suggested in the literature. Both Nagao et al. [8] and Niikawa et al. [11] proposed that the hematoma was exposed, diluted and washed out by CSF in the subarachnoidal space through a traumatically ruptured arachnoid membrane.

This washout theory seems likely in our case as the basal cisterns are filled on the followup CT. The blood which initially was subdural has (at least partially) probably transferred and mixed with CSF in the subarachnoidal space. The fact that the subdural hematoma in our case initially had a hyperdense signal intensity on CT, but remaining parts became isodense after only $4 \mathrm{~h}$ can be explained through the theory of dilution with CSF. This is also supported by the finding of a thin low-density area within the hematoma consistent with CSF in the subdural space. Such a low-density band was present in 22 of 28 cases (mainly adults) listed by Kato et al. [12]. However, a recent study that compared rapid resolution of ASDH with nonrapid resolution of ASDH found no difference in the presence of a low-density band [4]. 
Others speculated that, in addition to aforementioned CSF washout, acute brain swelling compresses the hematoma and leads to a superimposed early redistribution of the hematoma [13]. Wong et al. [9] reported about a 73-year-old patient with spontaneous regression of an ASDH in which they showed that blood was present in the spinal subdural space. Unfortunately, they did not have spinal MRI at the time of initial presentation, therefore it cannot be ruled out that the patient actually developed subdural hematoma at two distinct sites at one time due to the trauma.

\section{Conclusion}

The nature of this single case report limits firm conclusions on the management of ASDH in small children. We decided for the good neurological status to set a conservative approach without administration of medication or pressure monitoring. Others might have acted differently the moment the neurological status deteriorated slightly, and suspicion of an increased intracranial pressure was raised. The lesson that was learned was that our patient recovered fully without intervention, and it demonstrates that spontaneous resolution of ASDH can take place very fast, as observed by CT. Elucidating this mechanism further can perhaps identify patients in the future in whom acute operation is not needed.

\section{Statement of Ethics}

The family of the subject gave written informed consent to publish this case.

\section{Disclosure Statement}

The authors declare no conflicts of interest.

\section{Funding Sources}

The authors received no funding for this study.

\section{Author Contributions}

Hille Koppen drafted the paper and final version and collected the data. Enrico Arkink collected the data, contributed to the draft and the final version. Anna Nordbeck collected the data. Lilian Sie contributed to the draft and approved final version. Niels van der Gaag contributed to the draft and final version.

\section{References}

1 Wilberger JE Jr, Harris M, Diamond DL. Acute subdural hematoma: morbidity, mortality, and operative timing. J Neurosurg. 1991 Feb;74(2):212-8.

2 Gerard C, Busl KM. Treatment of acute subdural hematoma. Curr Treat Options Neurol. 2014 Jan;16(1):275. 
3 Lee KS, Bae WK, Bae HG, Doh JW, Yun IG. The computed tomographic attenuation and the age of subdural hematomas. J Korean Med Sci. 1997 Aug;12(4):353-9.

4 Brooke M, Patel A, Castro-Moure F, Victorino GP. Shedding new light on rapidly resolving traumatic acute subdural hematomas. J Surg Res. 2017 Nov;219:122-7.

5 Polman CH, Gijsbers CJ, Heimans JJ, Ponssen H, Valk J. Rapid spontaneous resolution of an acute subdural hematoma. Neurosurgery. 1986 Sep;19(3):446-8.

6 Kochanek PM, Carney N, Adelson PD, Ashwal S, Bell MJ, Bratton S, et al. Guidelines for the acute medical management of severe traumatic brain injury in infants, children, and adolescents - second edition. Pediatr Crit Care Med. 2012 Jan;13 Suppl 1:S1-82.

7 Evans JA, Bailey M, Vail A, Tyrrell PJ, Parry-Jones AR, Patel HC. A simple tool to identify elderly patients with a surgically important acute subdural haematoma. Injury. 2015 Jan;46(1):76-9.

8 Nagao T, Aoki N, Mizutani H, Kitamura K. Acute subdural hematoma with rapid resolution in infancy: case report. Neurosurgery. 1986 Sep;19(3):465-7.

9 Wong ST, Yuen MK, Fok KF, Yuen SC, Yam KY, Fong D. Redistribution of hematoma to spinal subdural space as a mechanism for the rapid spontaneous resolution of posttraumatic intracranial acute subdural hematoma: case report. Surg Neurol. 2009 Jan;71(1):99-102.

10 Huang SH, Lee HM, Lin CK, Kwan AL, Howng SL, Loh JK. Rapid resolution of infantile acute subdural hematoma: a case report. Kaohsiung J Med Sci. 2005 Jun;21(6):291-4.

11 Niikawa S, Sugimoto S, Hattori T, Ohkuma A, Kimura T, Shinoda J, et al. Rapid resolution of acute subdural hematoma-report of four cases. Neurol Med Chir (Tokyo). 1989 Sep;29(9):820-4.

12 Kato N, Tsunoda T, Matsumura A, Yanaka K, Nose T. Rapid spontaneous resolution of acute subdural hematoma occurs by redistribution - two case reports. Neurol Med Chir (Tokyo). 2001 Mar;41(3):140-3.

13 Matsuyama T, Shimomura T, Okumura Y, Sakaki T. Rapid resolution of symptomatic acute subdural hematoma: case report. Surg Neurol. 1997 Aug;48(2):193-6. 


\section{Case Reports in Neurology}
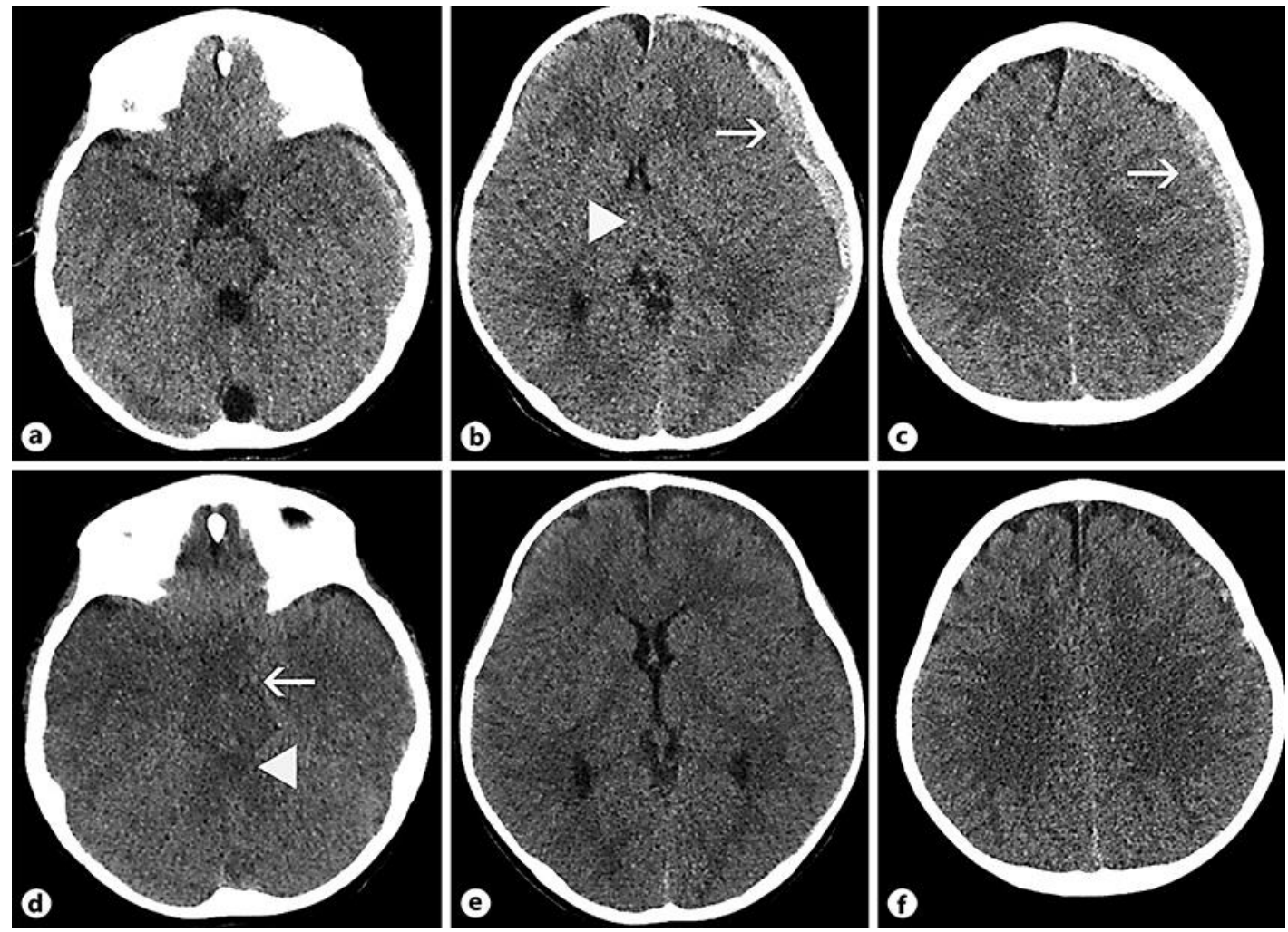

Fig. 1. a-c Nonenhanced CT scan at presentation. a Hyperdense, acute left convexity subdural hematoma on the left side. Basal cisterns are normal. b Left convexity subdural hematoma (arrow). Midline shift of $7 \mathrm{~mm}$ towards the right side (arrow head). c Subdural hematoma over left convexity (arrow). Note the small hypodense band on the inner margin of the hematoma, possibly indicating subdural CSF due to arachnoid tear. $\mathbf{d}-\mathbf{f}$ Follow-up CT acquired $4 \mathrm{~h}$ after presentation. $\mathbf{d}$ Increased density of the CSF in the basal cisterns (arrow), including the fourth ventricle (arrowhead). e Decrease of mass effect with near normalization of the midline shift. Subdural hematoma largely diminished in thickness. The density of the remaining subdural collection has become hypointense. f Some residual amount of hyperintense blood can still be seen over the left convexity. 